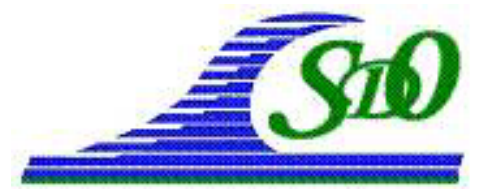

XI ìmes Journées Nationales Génie Côtier - Génie Civil

Les Sables d'Olonne, 22-25 juin 2010

DOI:10.5150/jngcgc.2010.010-D C Editions Paralia CFL

disponible en ligne - http://www.paralia.fr - available online

\title{
Développement d'un modèle opérationnel de prévision de houle à petite échelle sur le littoral transfrontalier des Pyrénées Atlantiques - Gipuzkoa
}

\author{
Jérémy DUGOR ${ }^{1}$, Didier RIHOUEY ${ }^{1}$, Fabrice ARDHUIN ${ }^{2}$
}

1. CASAGEC - ADERA, UFR Sciences et Techniques, 1 Allée du Parc Montaury, 64600 Anglet, France.

jeremy.dugor@univ-pau.fr

2. Ifremer, Centre de Brest, BP 70, 29280 Plouzané, France.

ardhuin@ifremer.fr

\section{Résumé :}

Dans le cadre du projet européen INTERREG IVa LOREA, CASAGEC a développé un modèle opérationnel de prévision de houle à petite échelle sur le littoral transfrontalier des Pyrénées Atlantiques - Gipuzkoa. Les données de houle issues du modèle serviront pour les diverses applications du projet qui vise à développer des outils de modélisation et de prévision pour les zones côtières et interrégionales. Le modèle SWAN, utilisé pour ce projet, est forcé aux frontières par les données spectrales fournies par le projet PREVIMER ainsi que par les données de vent du modèle GFS du NOAA/NCEP.

Cet article, qui vise à présenter le modèle et à analyser les premiers résultats, permet de valider la qualité de la modélisation. Les résultats sur le modèle interrégional sont satisfaisant avec des erreurs autour de $20 \%$ pour la hauteur significative moyennée sur 3 heures. Cependant, certains points faibles et des différences entre les modèles SWAN LOREA et les modèles PREVIMER ont été identifiés.

Mots-clés :

Prévision de houle - SWAN - WAVEWATCH IIITM - Modélisation opérationnelle Gipuzkoa - Pyrénées Atlantiques

\section{Introduction}

Le littoral transfrontalier des Pyrénées Atlantiques - Gipuzkoa occupe une position centrale au sein du Golfe de Gascogne qui favorise la dynamique démographique et le développement économique de ces $120 \mathrm{~km}$ de côte. De fait, ce littoral est soumis à une pression anthropique de plus en plus forte qui rend essentiel le développement d'outil d'aide à la décision permettant de connaître l'état du milieu et ainsi prédire l'arrivée et l'impact de pollution d'origine terrestre ou marine. Le projet européen INTERREG IVa LOREA (Littoral, Océan et Rivières d'Euskadi-Aquitaine) vise à développer des outils opérationnels d'observations, de modélisations et de prévisions pour deux échelles spatiales selon les applications : 
- L'échelle interrégionale, qui s'étend de la baie de Bilbao jusqu'au département des Landes, servira de base aux applications transfrontalières de transport de matériaux (hydrocarbures et déchets flottants).

- L'échelle locale, dont douze zones d'applications ont été définies en fonction des problématiques et des enjeux spécifiques (Qualité des eaux de baignade, sécurité des zones de bains, dynamique sédimentaire, arrivée de nappes d'hydrocarbures).

Dans le cadre de LOREA, CASAGEC est chargé de la mise en place d'un modèle de prévision de houle opérationnel qui doit être fonctionnel pour toutes les échelles du projet et donner des prédictions raffinées pour alimenter des modèles de circulation côtière. Le modèle SWAN (Simulating WAves Nearshore, BOOIJ et al., 1999), qui remplit ces conditions et permet de constituer des emboîtements de domaines, a été retenu.

Cet article reprend la stratégie mise en place pour modéliser la houle, une courte description du principe de fonctionnement du modèle opérationnel et une présentation des premiers résultats suivie d'une discussion sur les erreurs observées et les perspectives de travail envisagées.

\section{Modélisation des vagues}

\subsection{Choix du modèle}

SWAN, modèle spectral de houle de $3^{\text {ème }}$ génération a été paramétré pour implémenter la contribution du vent, les interactions entre triplets et quadruplets de fréquence et la dissipation d'énergie par déferlement (au large ou à la côte). Au regard de la configuration de la cote basque française (pas d'archipel d'îles, pas de pointes prononcées...), les gradients de l'état de la mer sont beaucoup moins importants que pour une côte découpée telle que la mer d'Iroise où PREVIMER utilise le modèle WAVEWATCH III, dans une configuration avec maillage non-structuré (ARDHUIN et al., 2009). Ici, le modèle converge en moyenne en moins de 15 itérations sans aide des limiteurs. A ce stade du projet et dans un souci de réduire les temps de calcul, les modules de diffraction, de dissipation par friction sur le fond, de set-up induit par les vagues n'ont pas été activés. Certains de ces modules pourront être appliqués par la suite en fonction des sites locaux et des applications connexes. Les calculs sont effectués en mode non stationnaire avec un pas de temps de 3 heures.

\subsection{Forçages}

Le modèle est forcé à ses frontières ouvertes par PREVIMER qui utilise plusieurs configurations multi-grilles, modèle WAVEWATCH IIITM basé sur le code de propagation de TOLMAN (2009) avec les paramétrages physiques de ARDHUIN et al. (2009). En pratique les spectres utilisés par SWAN sont issus du domaine Mer du Nord - Golfe de Gascogne à $0.03^{\circ}$ de résolution, emboîté dans un domaine global à $0.5^{\circ} \mathrm{de}$ 
résolution. Ces modèles PREVIMER sont forcés par les vents du Centre Européen de Prévision Météorologique à Moyen Terme (CEPMMT/ECMWF), de résolution $0.25^{\circ}$ toutes les 3 heures, et les courants barotropes du démonstrateur D1 de PREVIMER (modèle MARS2D). Depuis septembre 2008, ce système est le plus précis pour les prévisions de hauteur et périodes en Atlantique Nord-Est (BIDLOT, 2008). Ces spectres de houle sont fournis en 27 points espacés de $0.1^{\circ}$. Ces spectres, qui alimentent le modèle SWAN deux fois par jour pour 6 jours de prévisions, sont décomposés en 24 bandes de direction et 32 plages de fréquence allant de 0.037 à $0.716 \mathrm{~Hz}$ pour les deux modèles. Les données de vent sont issues du modèle GFS (Global Forecast System NOAA/NCEP). Les données sont récupérées deux fois par jours pour les 6 jours de prévisions avec un pas de temps de 3 heures. Notons que la résolution spatiale (figure 1) de ces prévisions $\left(0.5^{\circ}\right)$ est relativement grossière comparée à celle de la grille interrégionale $\left(0.01^{\circ}\right)$. Il est prévu dans le projet de récupérer les données des modèles de vent de l'institut Euskalmet avec une résolution plus fine. La hauteur du plan d'eau est fixe sur le modèle interrégional et intermédiaire. Sur les modèles côtiers, elle varie de façon statique sur les grilles toutes les 3 heures. Les marées sont calculées avec FES 2004.

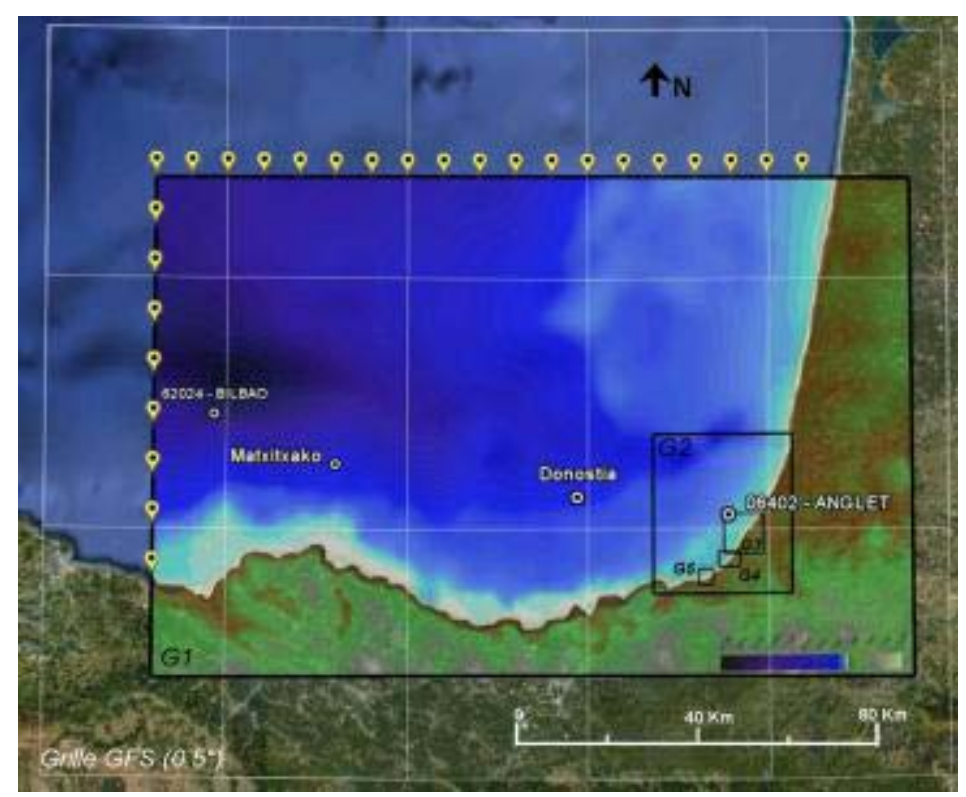

Figure 1. Configuration des grilles de calcul (cadres noirs). Situation des bouées de mesure de houle (points blancs). Points de données spectrales (points jaunes) et grille du modèle GFS pour l'implémentation du vent (traits fins blancs).

\subsection{Données bathymétriques et emboîtements}

Le modèle numérique de terrain pour chaque grille a été réalisé à l'aide des données du SHOM pour la partie océanique, de AZTI-Tecnalia pour la côte espagnole et par CASAGEC pour les données proches des côtes françaises. Les modèles numériques de 
terrain des zones côtières sableuses pourront être mis à jour en conséquence via le suivi topo-bathymétrique mis en œuvre par CASAGEC. Toutes les grilles sont rectangulaires et régulières. Un emboîtement de ces grilles à trois niveaux (figure 1) permet de faire la transition entre le modèle interrégional G1 $\left(\Delta=0.01^{\circ}\right)$ et les modèles à petite échelle $\mathrm{G} 3$, G4 et G5 $(30 \mathrm{~m}<\Delta<100 \mathrm{~m})$. La grille G2 $(\Delta=250 \mathrm{~m})$ sert d'intermédiaire.

\section{Principe de fonctionnement du modèle opérationnel}

Le principe de fonctionnement du modèle opérationnel peut se décomposer en trois étapes. Dans un premier temps, les données de prévisions de houle et de vent sont téléchargées via un protocole FTP et sont stockées dans une base de données. Cette opération est réalisée deux fois par jour (6h et 18h). Les fichiers d'entrée SWAN sont ensuite configurés pour chaque domaine avec les forçages correspondants (section 2.2). Une fois le calcul terminé, la troisième étape consiste à stocker les résultats et les afficher sur le site Internet. http://web.univ-pau.fr/CASAGEC/LOREA. Les résultats principaux sont également transférés vers le portail PREVIMER et aux partenaires du projet en charge de modéliser d'autres phénomènes à partir des données de houle.

Les résultats consultables sur Internet correspondent uniquement à la prévision. Les prévisions s'étalent sur une période de 6 jours avec un pas de temps de 3 heures. Chaque calcul contient une partie d'analyse (résultats se situant dans le passé). Cette partie analyse historique, stockée dans une base de données, permet de confronter les résultats du modèle avec les différentes mesures (section 4.2) afin de caractériser la validité des résultats. Caractériser les résultats des prévisions nécessite des méthodes statistiques différentes et une période de résultat plus étendue que la notre, cette partie ne sera pas abordée dans cet article.

\section{Validation des résultats}

Cette étude intervient seulement 3 mois après la mise en place du modèle et les données de mesures ne sont pas nombreuses à ce jour. Cette section vise à établir une première approche pour caractériser la validité des résultats face aux mesures et apprécier l'erreur engendrée par le modèle en lui-même ou par les forçages.

\subsection{Réseau de mesure}

Quatre bouées de mesure sont installées dans la zone du projet (tableau 1 et figure 1). Trois bouées situées en eau profonde et une sur le plateau continental à $50 \mathrm{~m}$ de profondeur et à près de $5 \mathrm{~km}$ des côtes. Les données de la bouée Bilbao, propriétés d'un institut espagnol extérieur au projet, ne sont pas disponibles à ce jour. Les trois autres bouées, situées dans la zone du modèle Interrégional, sont ici utilisées pour valider ce dernier. Les résultats du modèle intermédiaire diffère peu, ce modèle est surtout utile pour le passage de l'échelle océanique à l'échelle locale. Les zones des modèles locaux n'étant pas équipées d'instrument de mesures, aucune validation n'est encore possible. 
Tableau 1. Réseau de bouées de mesure concernant le projet LOREA.

\begin{tabular}{lllll}
\hline Station & \multirow{2}{*}{ Prof. $(\boldsymbol{m})$} & $\begin{array}{l}\text { Mis en } \\
\text { service }\end{array}$ & Fonctions & Propriétaire \\
\hline Bilbao & $\sim 600$ & Août 1990 & Vent, courant, salinité, vague directionnel & Puertos del Estado \\
Matxitxako & $\sim 450$ & Mai 2008 & Idem & DMC \\
Donostia & $\sim 550$ & Janvier 2007 & Idem & DMC \\
Anglet & $\sim 50$ & Déc. 2009 & Vagues directionnelles & LASAGEC \\
\hline
\end{tabular}

4.2 Validité du modèle

Les séries temporelles des hauteurs significatives des vagues pour les trois bouées disponibles (Matxitxako, Donostia et Anglet) sont représentées figure 2 pour le mois de décembre 2009 et indiquent les résultats des modèles SWAN et WAVEWATCH IIITM.

a)

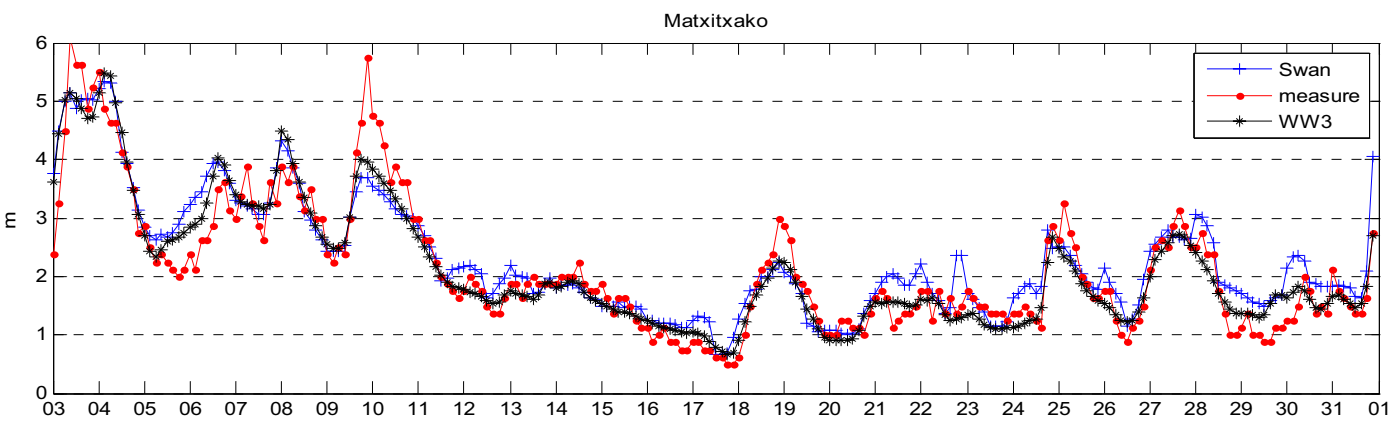

b)

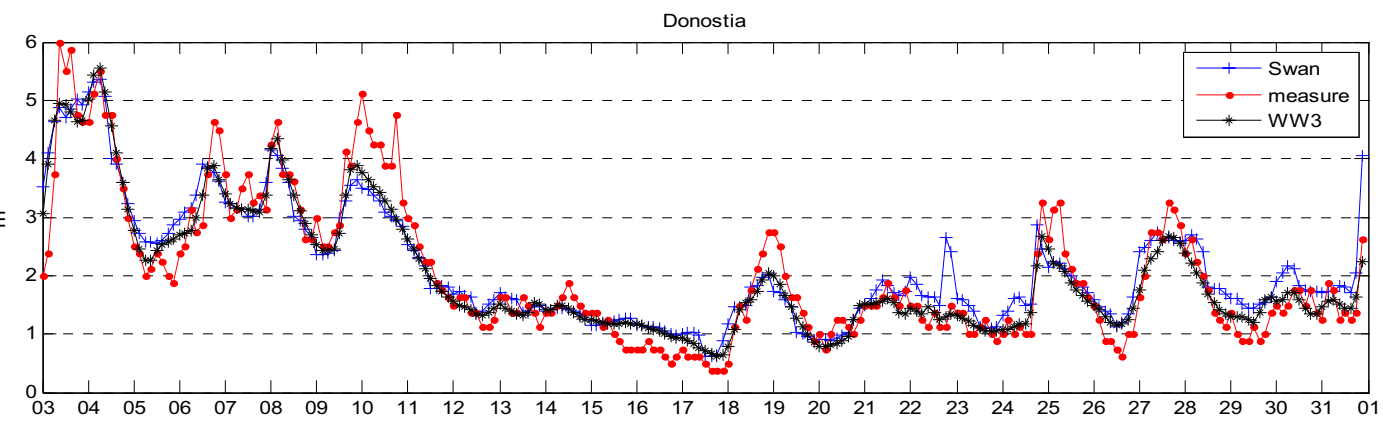

c)

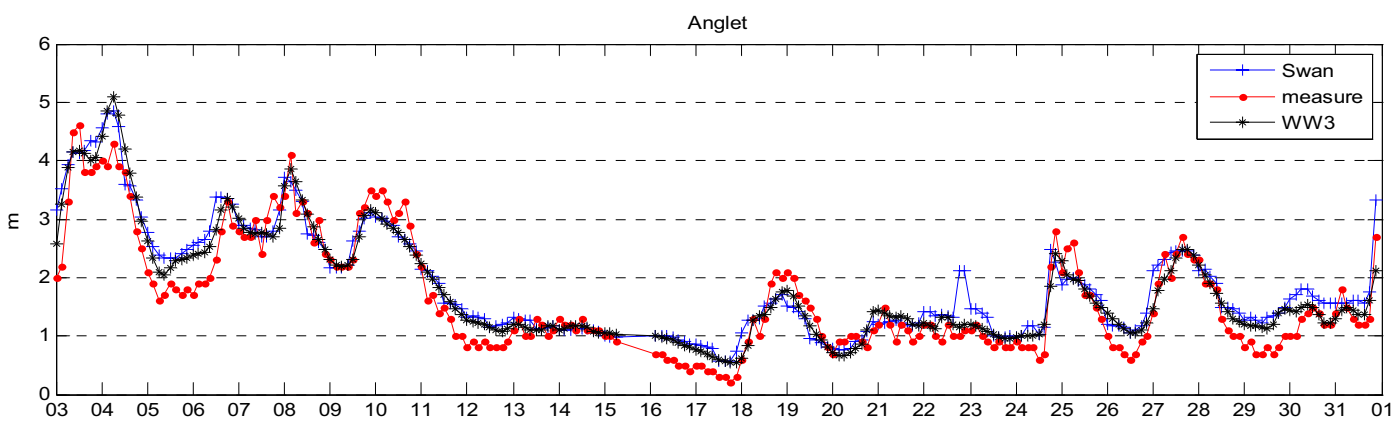

Figure 2. Séries temporelles (décembre 2009) des hauteurs significatives des vagues pour le modèle SWAN (bleues), les bouées (rouges) Matxitxako (a), Donostia (b) et Anglet (c) et pour le modèle WAVEWATCH III TM PREVIMER (noires). 
Les indices des erreurs statistiques de ces résultats sont représentés dans le tableau 2. Les résultats sont satisfaisants avec des coefficients de corrélation supérieurs à 0.9 et un Scatter Index autour de 20\%. Les biais sont négatifs et proche de zéro, ce qui indique une tendance à la surestimation des modèles. Les résultats du modèle WAVEWATCH $\mathrm{III}^{\mathrm{TM}}$ sont légèrement meilleurs que ceux de SWAN. En ce qui concerne le vent, la différence relative des vitesses $U_{10}$ entre les modèles ECMWF et GFS est de $23 \%$ avec un biais de $0.2 \mathrm{~m} / \mathrm{s}$. En ramenant ces vitesses (WU, 1982) à la hauteur de mesure de la bouée Donostia ( $3 \mathrm{~m}$ ), ECMWF et GFS présentent respectivement des NMRSE de 32\% et $34 \%$ avec des biais de 1.06 et $0.89 \mathrm{~m} / \mathrm{s}$.

Tableau 2. Erreurs des hauteurs significatives des modèles SWAN et WAVEWATCH III ${ }^{\mathrm{TM}}$ aux bouées Matxitxako, Donostia et Anglet durant décembre 2009.

\begin{tabular}{|c|c|c|c|c|c|c|}
\hline \multirow{2}{*}{ Erreurs sur le HS } & \multicolumn{2}{|c|}{ Matxitxako } & \multicolumn{2}{|c|}{ Donostia } & \multicolumn{2}{|c|}{ Anglet } \\
\hline & $S W A N$ & $W W 3$ & $S W A N$ & $W W 3$ & $S W A N$ & $W W 3$ \\
\hline BIAIS (m) & -0.14 & 0.01 & -0.07 & 0.04 & -0.20 & -0.13 \\
\hline$R M S E(m)$ & 0.49 & 0.37 & 0.51 & 0.39 & 0.41 & 0.33 \\
\hline NRMSE (\%) & 23 & 17 & 25 & 19 & 24 & 20 \\
\hline SI $(\%)$ & 21 & 17 & 25 & 19 & 23 & 19 \\
\hline Coef. de corrélation & 0.91 & 0.94 & 0.91 & 0.95 & 0.93 & 0.95 \\
\hline
\end{tabular}

Durant la période d'observation du mois de décembre 2009, des évènements de houle sont plus ou moins bien modélisés. Trois types d'erreurs ont pu être mises en évidence.

- Différences observées entre les deux modèles :

Pour plusieurs jours d'observations, les résultats du modèle SWAN diffèrent de

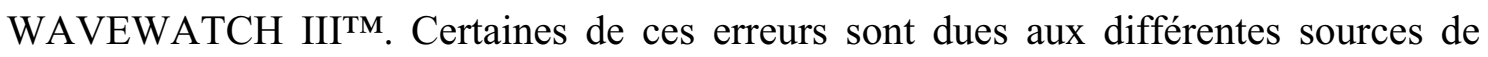
vent implémentées (section 2.2), par exemple pour le 22/12/09 à 18h TU, la vitesse du vent GFS ramené à la hauteur de la bouée indique $11.5 \mathrm{~m} / \mathrm{s}$ contre $4.2 \mathrm{~m} / \mathrm{s}$ pour la bouée à Donostia. Dans d'autres cas, par exemple le 17/12/2009, les deux sources de vent sont cohérentes avec la mesure mais SWAN surestime la hauteur des vagues pour les deux bouées en eaux profondes (Matxitxako et Donostia). Dans ce cas, ainsi que le 22 décembre, une houle longue de période $14 \mathrm{~s}$ et hauteur autour de $1 \mathrm{~m}$ est prévue. La surestimation de SWAN est typique des erreurs du paramétrage WAMDI (1988) en présence de houle: la dissipation de mer du vent est artificiellement réduite ce qui aboutit à une hauteur significative trop importante. Ce problème existe encore avec d'autres paramétrages (voir ARDHUIN et al., 2007), et il n'a été supprimé que dans les paramétrages de TOLMAN et CHALIKOV (1996), VAN DER WESTHUYSEN et al. (2007) et ARDHUIN et al. (2009).

- Sous-estimation des modèles des entrées de houle énergétiques et rapides :

Lors des évènements du 10, du 19 et du 25 décembre 2009 (figure 2), les pics de houle mesurés aux trois bouées sont sous-estimés par les deux modèles. L'entrée de houle est 
courte mais énergétiques pour ces trois jours observés. La différence de hauteur significative modélisée et mesurée tend à s'atténuer lorsqu'on s'éloigne des frontières de forçages. Les données de vent mesurées pour ces évènements indiquent que le vent local est bien modélisé et n'est donc pas la cause de ces erreurs.

- Surestimation des modèles pour des vents de terre:

Les modèles surestiment souvent la mesure pour les conditions de vent de terre (figure 2 : observation pour les évènements du $6,12,17,24,26$ et 29 décembre 2009). Pour tous ces évènements, les vents sont de secteur Sud à Est Nord-est, soit un vent de terre. Cela a pour effet de faire diminuer la taille de la houle, la période et dans la plupart des cas la direction moyenne varie. Cette décroissance de la houle est surestimée par les deux modèles et le phénomène est d'autant plus marqué à la bouée côtière d'Anglet. Les vents modélisés sont similaires à la mesure et donc ne sont pas la source de ces erreurs. Dans le cas de PREVIMER, il est possible que la résolution spatiale du modèle $(4 \mathrm{~km}$ pour le modèle lui-même et $25 \mathrm{~km}$ pour le vent utilisé) soit insuffisante en regard de la distance à la côte. Pour le modèle SWAN, il est probable que le développement des vents de la côte vers le large ne soit pas résolu convenablement.

\section{Conclusions et perspectives}

Un modèle de prévision de houle sur le littoral transfrontalier des Pyrénées Atlantiques - Gipuzkoa, développé dans le cadre du projet européen LOREA, est opérationnel depuis septembre 2009. Cette étude, qui vise à présenter le modèle et à analyser les premiers résultats, permet de valider la qualité de la modélisation et de mettre en évidence des points faibles et des différences entre les modèles SWAN LOREA et PREVIMER :

(1) La qualité des prévisions est moyenne, typiquement moins bonne que pour la mer d'Iroise ou le Golfe du Lion, et comparable à la mer Ligure. Cela peut être lié au relief important proche des côtes, qui limite la qualité des vents modélisés.

(2) Lors d'une entrée de houle rapide et intense, les deux modèles sous estiment le pic de houle. La diminution du pas de temps à $1 \mathrm{~h}$ au lieu de $3 \mathrm{~h}$ pourrait améliorer la simulation de ces événements. Cependant, cela implique des forçages de vents échantillonnés à une $1 \mathrm{~h}$ et un temps de calcul plus élevé.

(3) Les modèles ont tendance à surestimer la hauteur de houle lorsque les vents passent aux secteurs Sud à Est Nord-est (vent de terre). Ce phénomène étant plus important au niveau de la bouée côtière d'Anglet. Ce genre de biais peut probablement être corrigé par une calibration du modèle SWAN, ou une adaptation statistique des résultats.

(4) L'implémentation d'un forçage de vent issu d'un modèle plus raffiné, prévue dans le cadre du projet LOREA, pourrait améliorer les résultats.

En termes de perspectives, outre les axes de travail énoncés précédemment, notons que l'analyse des résultats a été réalisée sur une durée de 1 mois (décembre 2009). La validation des résultats sur une période plus longue et des mesures en zone proche 
côtière permettra de mieux valider puis d'optimiser les prévisions à l'échelle interrégionale et locale.

\section{Remerciements :}

Ce travail a été réalisé au sein du projet européen INTERREG IVa LOREA financé dans le cadre du projet POCTEFA par des fonds FEDER. Nous tenons à remercier les partenaires du projet dont le co-financeur, le département des Pyrénées Atlantiques. Nous voudrions également remercier la collaboration avec les partenaires du projet PREVIMER (IFREMER et SHOM) ainsi que les partenaires espagnols pour la fourniture des données de houle (Meteorology and Climatology Direction of the Basque Government) et bathymétrique (AZTI Tecnalia).

\section{Références bibliographiques}

ARDHUIN F., HERBERS T.H.C., WATTS K.P., VAN VLEDDER G.P., JENSEN R., GRABER H. (2007). Swell and slanting fetch effects on wind wave growth. J. Phys. Oceanogr., vol. 37, no. 4, pp 908-931. doi:10.1175/JPO3039.1

ARDHUIN F., MARIE L., RASClE N., FORGET P., ROLAND A. (2009). Observation and estimation of Lagrangian, Stokes and Eulerian currents induced by wind and waves at the sea surface. J. Phys. Oceanogr., vol. 39, no. 11, pp 2820-2838. doi:10.1175/2009JPO4169.1

BIDLOT J.-R. (2008). Intercomparison of operational wave forecasting systems against buoys: data from ECMWF, MetOffice, FNMOC, NCEP, DWD, BoM, SHOM and JMA, September 2008 to November 2008. tech. rep., Joint WMO-IOC Technical Commission for Oceanography and Marine Meteorology.

BOOIJ N., RIS R.C., HOLTHUIJSEN L.H. (1999). A third-generation wave model for coastal regions. 1. Model description and validation. J. Geophys. Res., 104, C4, pp 7649-7666. doi:10.1029/98JC02622

TOLMAN H.L., CHALIKOV D. (1996). Source terms in a third-generation wind wave model. J. Phys. Oceanogr., vol. 26, pp 2497-2518. doi:10.1175/1520-0485(1996)026<2497:STIATG $>2.0 . C O ; 2$

TOLMAN H.L. (2009). User manual and system documentation of WAVEWATCH III version 3.14. NOAA / NWS / NCEP / MMAB Technical Note 276, 194 p.

VAN DER WESTHUYSEN A.J. (2007). Advances in the spectral modelling of wind waves in the nearshore. Ph.D thesis, Delft University of Technology, $206 \mathrm{p}$.

WAMDI Group (1988). The WAM model - a third generation ocean wave prediction model. J. Phys. Oceanogr. 18, pp 1775-1810.

WU J. (1982). Wind-stress coefficients over sea surface from breeze to hurricane. J. Geophys. Res., 87, C12, pp 9704-9706. doi:10.1029/JC087iC12p09704 